livraisons

d'Histoire

de l'Architecture

\section{Livraisons de l'histoire de l'architecture}

16 | 2008

Grands chantiers et matériaux

\title{
Fraudes et profits autour des marbres méridionaux, de Colbert au duc d'Antin
}

Fraud and profits by French southern marbles, from the age of Colbert to the duc d'Antin

Schmuggelgeschäfte und Profit in Betreff südländischen Marmors, von Colbert zum Herzog von Antin

\section{Pascal Julien}

\section{OpenEdition}

\section{Journals}

Édition électronique

URL : http://journals.openedition.org/lha/179

DOI : $10.4000 /$ lha. 179

ISSN : 1960-5994

Éditeur

Association Livraisons d'histoire de l'architecture - LHA

Édition imprimée

Date de publication : 10 décembre 2008

Pagination : 33-46

ISSN : 1627-4970

Référence électronique

Pascal Julien, «Fraudes et profits autour des marbres méridionaux, de Colbert au duc d'Antin », Livraisons de l'histoire de l'architecture [En ligne], 16 | 2008, mis en ligne le 10 décembre 2010, consulté le 19 avril 2019. URL : http://journals.openedition.org//ha/179 ; DOI : 10.4000//ha.179

Ce document a été généré automatiquement le 19 avril 2019

Tous droits réservés à l'Association LHA 


\section{Fraudes et profits autour des marbres méridionaux, de Colbert au duc d'Antin}

Fraud and profits by French southern marbles, from the age of Colbert to the duc d'Antin

Schmuggelgeschäfte und Profit in Betreff südländischen Marmors, von Colbert zum Herzog von Antin

Pascal Julien

« Il y a eu cy devant un très grand abus, même de

la prévarication dans le transport des marbres "

1 La fourniture de matériaux pour de grands chantiers s'avéra un problème d'une grande complexité dans le cas des marbres, blancs ou de couleur. Rares et recherchées, ces roches ornementales étaient difficiles à exploiter, malaisées à transporter et souvent très éloignées des sites de mise en œuvre. En dépit de ces inconvénients, leur caractère symbolique et leur valeur esthétique furent tels aux yeux de Louis XIV que les Bâtiments du roi redoublèrent d'effort pour s'en procurer, afin d'en parer le château et les jardins de Versailles. La politique d'approvisionnement qui fut alors mise en place et qui donna des résultats incomparables, a fait l'objet de plusieurs études ${ }^{1}$. Il convient toutefois de revenir sur ce sujet en l'abordant sous un jour différent. En effet, aux classiques questions de coût, de qualité, de quantité ou de disponibilité qui se posent en pareil cas, il faut ajouter un paramètre rarement pris en compte dans l'étude des chantiers mais pourtant séculaire et essentiel : celui des manœuvres, des fraudes et des profits bien compris. Un paramètre qu'il est toujours difficile, bien évidemment, de mettre en lumière, mais qui eut, dans le cas des marbres royaux, une ampleur et des conséquences non négligeables, à la hauteur des sommes vertigineuses mises en jeu. 


\section{Les excès d'une « société désimée »}

2 Depuis Henri II, les marbres de couleur français avaient acquis leurs lettres de noblesse auprès des Bâtiments du roi, car ils ajoutaient à d'indéniables qualités techniques et visuelles l'intérêt de concurrencer artistiquement et économiquement l'Italie, fournisseur traditionnel en la matière. Il en naquit une réelle notion d'orgueil national attachée à l'exploitation du sol du royaume, ce dont témoignent de nombreux textes. Cette notion ne fut pas étrangère à la volonté constante et déterminée de Colbert puis de Louvois de développer ces ressources par l'intermédiaire de marchands puis de «sociétés », en dépit de bien des difficultés. Les marbres du Midi ne furent pas les seuls sollicités, loin de là, et l'on continua de s'approvisionner en Flandre et en Hainaut ainsi qu'en Ligurie et en Toscane comme par le passé. Mais le développement du circuit français nécessita des interventions et adaptations successives qui demeurent consignées dans des archives d'une incroyable richesse, où transparaît parfois le dessous des cartes.

Dans un premier temps, Colbert proposa l'exploitation des sites du royaume à des fournisseurs qu'il mit en concurrence. En 1664,

Louis XIV, roi de France

5 Formont, Pierre, banquier

2

6 Banquiers et négociants traitaient directement avec les carriers et les convoyeurs, se chargeant de toutes les phases de la production et du transport jusqu'à Paris où ils étaient directement payés par les services des Bâtiments du roi. Entre 1668 et 1683, malgré une quasi-interruption du trafic durant la guerre de Hollande - de 1672 à 1679 - près de deux millions et demi de livres furent dépensées en achat de marbres de tous les pays; Formont

Formont, Pierre, banquier

8 Le grand avantage pour Colbert fut de déléguer ainsi toutes les opérations, confiées à des entrepreneurs spécialisés, déjà aguerris par le commerce avec l'Italie. Mais ce système avait aussi ses inconvénients : Formont n'eut de cesse que d'éliminer ses rivaux et parvint presque à un monopole qui lui laissait pratiquer des prix dont la pertinence était incontrôlable ; tout cela au risque de donner priorité à la quantité, au détriment de la qualité. Aussi le surintendant chargea-t-il Étienne Ducrot, en 1679,

Ducrot, Etienne, commis du roi 4

Louvois

11 Louvois, marquis de, surintendant des Bâtiments du roi

12 Dès le 12 juillet 1684 , il fit procéder à « l'adjudication du tirage et transport de tous les marbres dont le roi peut avoir besoin " à Caunes et le 20 août, il rééditait la même opération à Bagnères-de-Bigorre, pour les carrières de Campan et de Sarrancolin. Les affichages et proclamations "à sons de trompe " avaient été faits dans de nombreuses villes et des marbriers et tailleurs de pierre locaux, mais qui venaient aussi de l'ensemble du royaume, $\mathrm{y}$ participèrent. Ce fut finalement la même compagnie qui emporta les 
marchés des deux sites, tant d'extraction que de transport. Formée pour l'occasion dès le 28 avril de cette même année, elle était composée de Claude Le Seure, sieur de Chantemerle

Chantemerle, Claude Leseure, sieur de,

Chausset, Antoine

Cette compagnie, dite Chantemerle

Chantemerle, Claude Leseure, sieur de,

Chantemerle, Claude Leseure, sieur de,

Martin ; Michel, ingénieur du roi

67

Fondée sur de nouvelles bases, l'exploitation pour le roi s'était révélée des plus efficaces en quantité, mais aussi, apparemment, en coûts : alors que l'on payait Formont de 18 à 30 livres le pied cube rendu à Paris, selon la taille des blocs ${ }^{8}$, on réglait seulement 7 livres et 18 sous pour les marbres du Languedoc et des Pyrénées débarqués près de Versailles et 13 sous de plus jusqu'à Paris.

Toutefois, il subsiste bien des zones d'ombre pour cette période. Tout d'abord, l'origine de la compagnie Chantemerle

Chantemerle, Claude Leseure, sieur de,

Martin ; Michel, ingénieur du roi

Martin ; Michel, ingénieur du roi

En vérité, ce système fut bien moins rentable qu'il n'y parait. Le déroulement des enchères, voulu ou subi, avait induit des coûts de production et de transport irréalistes qui eurent des conséquences d'autant plus fâcheuses que, de 1692 à 1697, la longue guerre de la ligue d'Augsbourg interrompit tout trafic et donc toute livraison et tout paiement. D'une part, cela poussa certains des «associés » à se livrer à un commerce parallèle afin d'arrondir leurs gains. D'autre part, et surtout, ces faibles prix de revient eurent une conséquence directe sur les matériaux eux-mêmes.

L'exploitation des marbres demande une experte gestion du temps. Une carrière nécessite des soins attentifs et continus, sur le long terme, afin de pratiquerF des dégagements - ou découverts - suffisants et bien ciblés pour non seulement parvenir aux bonnes veines mais encore pouvoir suivre celles-ci au mieux et en profiter longuement. Lorsqu'ils prirent en main les carrières des Pyrénées, les ouvriers francs-comtois embauchés par la compagnie Chantemerle n'eurent qu'à approfondir les fronts de taille laissés par les carriers de Fromont qui, lui, n'avait pas hésité à investir dans de coûteux dégagements de roches inutiles. Mais à Sarrancolin comme à Campan, variétés les plus prisées par le roi, les bonnes veines plongeaient dans la roche, ce qui nécessitait de constants et difficiles découverts (ill. 1). 
III. 1 : Carrière de Veyrède (Hautes-Pyrénées)

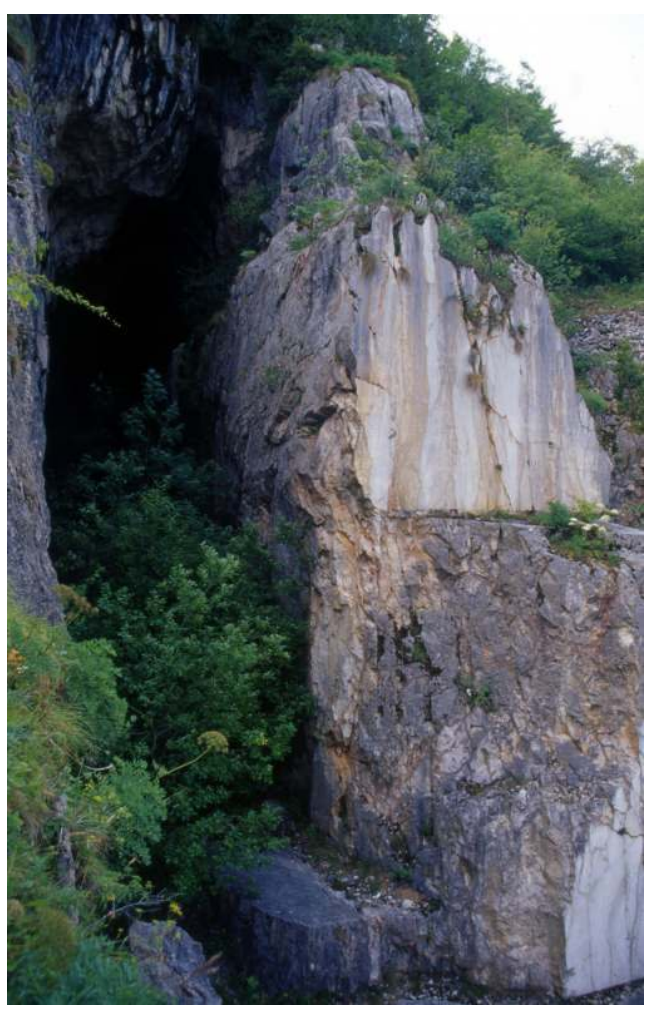

Des sciages modernes laissent apparaître un marbre de moyenne qualité. La bonne veine, elle, s'enfonçait dans la montagne et a nécessité le creusement de cette haute galerie, datant du XVIIIe siècle

Cl. P. Julien

Or ceux-ci avaient été exclus des marchés, lors des soumissions, ce qui avait permis de baisser les prix, mais les Bâtiments du roi se refusèrent très rapidement à les financer. Dès 1686, les filons furent épuisés à Sarrancolin et, malgré quelques coûteux travaux, ils le furent également en 1688 à Campan. Les entrepreneurs se refusèrent à opérer de nouveaux dégagements à leurs seuls frais, d'autant que leurs meilleurs ouvriers, écœurés par le mauvais climat et la dureté des marbres pyrénéens, avaient préféré se replier sur Caunes en Minervois, où les sites d'incarnat et de turquin étaient bien mieux exposés et leurs veines à ciel ouvert. En conséquence, la compagnie Chantemerle se résolut rapidement à ne plus exploiter que ces rouges-là, dont ils inondèrent les magasins du roi : ils en livrèrent quatre fois plus que des autres variétés, et il en demeura en carrière et dans les ports une quantité presque égale à convoyer.

Les déboires de la compagnie Chantemerle donnèrent lieu à un procès en 1692 à la bourse des marchands de Paris avec le «riche banquier » parisien Jean Dubois, qui gérait une partie des sommes versées par les trésoriers royaux. Ce litige, qui nécessita l'intervention directe du surintendant Colbert de Villacerf, précipita la fin de l'association des entrepreneurs qui s'accusèrent mutuellement d'erreurs, de ventes illicites ou de détournements, car certaines sommes avaient été avancées avant livraison ${ }^{10}$. L'un d'eux, Petit, qui avait truqué ses livres de compte et probablement vendu des rouges du Languedoc à des marchands italiens, fut même emprisonné à Toulouse, accusé d'avoir «mangé et consummé » pour "bien se divertir avec ses amis » une partie de l'argent perçu pour les transports ${ }^{11}$. Toute l'organisation finit par faire faillite et Martin fut 
extrêmement soulagé, en 1692, de se voir nommé à Antibes, car il subissait continuellement « les persécutions des créanciers » de cette " société désimée »"

L'opacité du système mis en place était telle qu'il fut impossible, pour les Bâtiments du roi de quantifier avec précision les quantités produites et livrées. Ainsi à la suite de quelques transports réalisés après 1697 et la paix de Ryswick, le compte définitif de la compagnie Chantemerle dressé

Chantemerle, Claude Leseure, sieur de,

Cotte, Robert de, architecte

1314

En ce qui concerne les carrières, en 1692, Colbert de Villacerf avait fait « réserver pour le roy " celles de Caunes, de Campan et de Sarrancolin sur les conseils de Martin afin d'éviter, durant la guerre qui interrompait les travaux, que n'y soit enlevé par des particuliers ce qu'il y restait de beaux marbres. En dépit de ces interdictions, ce fut la ruée et même les surveillants nommés par les intendants se permirent de tirer « impunément » du marbre. À Caunes pour exemple, la « belle marbrière » où l'on taillait de hautes colonnes, telles celles du Grand Trianon, fut prise d'assaut et le surintendant lui-même

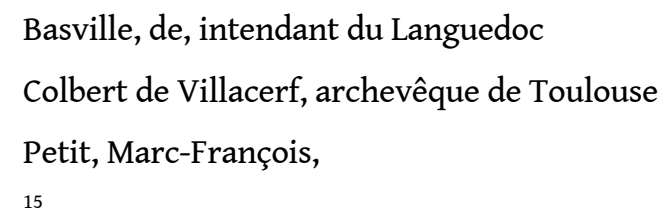
magasins d'un certain nombre de blocs de moyenne voire de médiocre qualité, fruits du système des compagnies. En dépit de ses efforts, l'accumulation de rouges de Languedoc et de marbres de rebut n'alla pourtant qu'en augmentant avec les dernières livraisons méridionales. En 1699, Jules Hardouin-Mansart, devenu surintendant, chercha à son tour à y remédier. Afin de vendre les stocks accumulés, il institua un contrôle du transport des marbres et il en fit réserver le commerce au roi, par un arrêt de 1701. Un tel acte instituait un monopole commercial en faveur des Bâtiments du roi, qui pouvaient désormais se défaire à des prix élevés de leurs pierres de second ordre, voire de rebut, ce qui allait développer un fort marché parallèle. Son successeur allait pleinement profiter de cette situation.

\section{Les profits du duc d'Antin}

La politique marbrière supervisée par Colbert avait essentiellement suscité des oppositions, manœuvres et coups bas entre négociants, sans grande conséquence sur la qualité des livraisons, alors que la politique menée par Louvois avait entraîné une faillite financière et un déséquilibre des variétés stockées en magasin, pour certaines fort médiocres. La guerre empêcha Hardouin-Mansart de mieux organiser cet approvisionnement et ce fut le duc d'Antin, à partir de 1712, qui réussit à l'optimiser vraiment, en créant un département des marbres des plus efficaces. Toutefois, l'intérêt qu'il porta à ce matériau fut très largement amplifié par une implication très personnelle dans son trafic. 


\section{2 : Détail de la Carte des Montagnes et vallées d'Aure [...] Jusques au dessous de Montespan, par} Hippolyte Matis, 1719

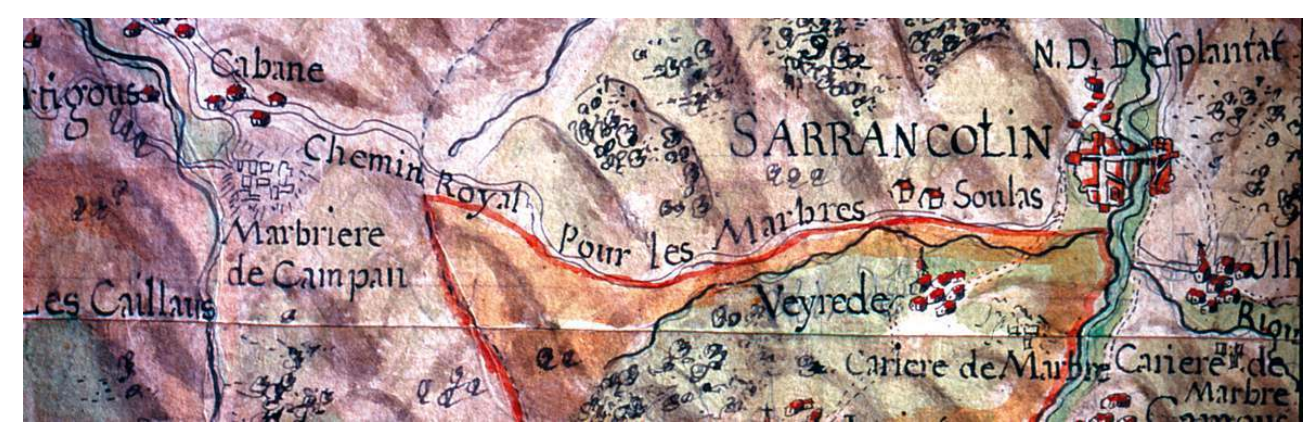

Arch. départ. Yvelines, A 404. Les terres et forêts du duc d'Antin, entourant la carrière de Veyrède, sont cernées d'un trait épais. Elles sont longées par le nouveau chemin royal des marbres, allant de la marbrière de Campan à Sarrancolin

Cl. P. Julien son marquisat et le contrôleur des marbres, lui proposa de créer un chemin entre cette carrière de Campan et le port de Sarrancolin, où l'on chargeait les marbres sur des radeaux, ce qui éviterait les longs, coûteux et difficiles charrois par la vallée de l'Adour. Le duc d'Antin l'y encouragea et ajouta : « Vous vous servirez le plus possible de mes bois pour faire les radeaux et s'il y a quelque dépense à faire pour tirer lesdits bois vous la ferez en mesme temps que le chemin ${ }^{17}$. » Tout était dit : les bois du duché d'Antin allaient bientôt être coupés gratuitement et flotter jusqu'à Bordeaux tout aussi gratuitement, car depuis le privilège octroyé en 1670 et renouvelé en 1684, tout chargement de ce type était exonéré de droits. De plus, les chemins de débardage de ses forêts allaient être discrètement mais intégralement financés par les Bâtiments du roi. Il en confia même le tracé à un ingénieur de l'intendance de Languedoc.

Le chemin fut en grande partie réalisé dès 1713 puis un autre suivit, depuis d'autres forêts du duc d'Antin. Celui-ci intervint également auprès de la chambre des Eaux et forêts du parlement de Toulouse pour améliorer la navigation ${ }^{18}$. De telles mesures n'étaient pas sans intérêt pour faciliter le flottage des grands fûts, les plus rentables. En 1714, il écrivait 
à Lassus : "Envoyez moi le nom de mes bois pour que je fasse expédier par M. de Pontchartrin une permission pour les couper et que personne ne puisse troubler les vendeurs ny acquéreurs, j'espère en tirer un profit considérable ${ }^{19}$. » La permission ayant été délivrée par le ministre de la Marine, seul compétent en matière de hautes futaies susceptibles de fournir des mâts de bateau, Lassus fit couper les bois pressentis dans les forêts du duc, les fit évacuer par les chemins de débardage ouverts aux frais du roi puis en fit confectionner des radeaux. Ceux-ci étant arrivés au terme de leur voyage, en 1714, le duc fit signifier par Lassus à son protégé Aziron, inspecteur des marbres à Bordeaux :

De vandre à mesure que les bois arriveront et de les vandre leur prix, d'en emploier le produit aux dépences qu'il est obligé de faire pour les marbres, de vous en envoier les comptes en bonne forme pour me faire rembourser icy [...] les première pièces sont toujours difficiles à venir, j'espère à présent tirer un produit raisonnable des marbres de vos pirénées par vos soins et vos arrangements ${ }^{20}$.

Il évitait ainsi de gênants dépôts et transferts d'argent. Il n'hésita pas à mobiliser plus encore les services royaux et en 1716 il écrivait à Pierre de Lassus :

J'envoye en Guyenne le sr Matis arpenteur du roy, homme très habile et en qui j'ay grande confiance pour lever le cour de la Garonne, toutes les carrières de marbre des Pyrénées, les nouveaux chemeins et tout ce que vous croirez nécessaire pour le service, il lévera en même temps toutes mes terres, vous verrez combien l'ouvrage est beau et utile ayant fait ainsi dans toutes mes autres terres (ill. 2) ${ }^{21}$.

Une fois exécutées, ces cartes effectivement magnifiques, de plus de 14 mètres de long, lui servirent à mieux situer ses possessions et de possibles coupes de bois, mais aussi à repérer tous les problèmes de transport afin de faire rectifier les cours d'eau, jusqu'à Bordeaux.

Mais le duc d'Antin sut encore pousser son avantage. Dès avril 1715, il avait organisé une présentation de marbres méridionaux pour le roi qui « les trouva fort beaux et toute la cour qui les examina longtemps. Le veirède emporta le prix, dont je suis fort aise $»^{22}$. Dès lors, ce sarrancolin allait prendre le nom de "marbre d'Antin », qu'il a conservé. Très finement, le duc fit savoir au roi que ce marbre lui appartenait mais qu'il le cédait avec grand plaisir à la couronne, renonçant à son droit de fortage ${ }^{23}$. En contrepartie, il obtint de pouvoir faire venir des marbres pour son propre usage, ainsi qu'il l'exprima en 1727, lorsqu'il sollicita la reconduction de ce privilège auprès de Louis XV :

Le duc d'Antin supplie votre Majesté de vouloir bien lui continuer la permission qu'il a toujours eue de faire venir des Pyrénées avec les marbres de votre Majesté, des marbres pour son usage, d'autant qu'il ne retire aucune rétribution de ceux qu'il fournit pour les Bâtiments, ni des bois qui servent aux radeaux ${ }^{24}$.

50 L'allusion aux pertes sur le bois est ici des plus savoureuses. Ce privilège fut très pratique pour faire venir des blocs à Paris, aux frais du roi encore, blocs qui furent très probablement débités et vendus par Tarlé, qui avait très étrangement obtenu une «permission de monseigneur le duc d'Antin pour l'achat et débit » du marbre. Mais d'autres sources alimentaient ce trafic.

51 En 1737, à Bordeaux, l'architecte Gabriel fut avisé du fait « qu'il y a eu cy devant un très grand abus même de la prévarication dans le transport des marbres » et des ventes illicites opérées à Bordeaux par l'ancien directeur des Bâtiments du roi lui furent signalées, mais cela resta sans suite ${ }^{25}$. Il y en eut toutefois un écho en 1749 , quand la complexe succession de l'un des membres de la compagnie Chantemerle déboucha sur la plainte d'un particulier, qui réclama six blocs demeurés sur le port depuis plus de soixante ans et jamais payés par les Bâtiments du roi. L'intendant de Tourny, qui à cette 
occasion fit faire des vérifications dans les inventaires des marbres du port, eut la surprise d'y découvrir des états contrefaits datant de 1715, Tarlé ayant antidaté et signé des papiers que d'Antin visait en blanc. Le directeur Lenormant de Tounehem, interrogé sur la nécessité d'une enquête, s'empressa de classer l'affaire et d'ordonner l'indemnisation immédiate de l'héritier, dont l'affaire traînait pourtant depuis des années :

La contradiction qui se trouve dans les dates des certificats de feu M. le duc d'Antin et du sr Tarlé [...] mériteroit une attention particulière, s'ils n'estoient accompagnés de toutes les autres marques de la vertu, comme la signature de Mr le duc d'Antin, du sceau de ses armes et de la contre signature du secrétaire des Batiments du roi avec la signature du sr Tarlé. Mais il y a tout lieu de croire que la faute provient d'un vice de clerc ${ }^{26}$.

52 Toutes les marques de la vertu! En fait il semble que, durant des années, sous couvert d'agir pour le roi, le duc ait fait évacuer des blocs restés en souffrance à Bordeaux depuis la surintendance de Louvois. Il y en avait de deux sortes : ceux de rouge de Languedoc qui étaient restés stockés sur les atterrissements, faute d'être portés à Paris où ils étaient en surnombre mais aussi ceux, de toutes variétés, qui étaient envasés dans le port et qui n'avaient jamais été inventoriés, sauf par...Tarlé, en 1715 ! Ils étaient très nombreux, par la faute de radeliers impatients qui, souvent, s'en débarrassaient à leur arrivée à marée basse au port, encore loin des quais. Dans la seconde moitié du siècle, l'intendance en fit retirer plus de 230, avec une quinzaine de colonnes. Mais des dévasements plus discrets furent également organisés par le duc, comme en 1723 où des ouvriers furent « employés à sortir et déterrer des blocs de marbre " et à les charger sur des bateaux en partance pour le Havre ${ }^{27}$. Échappant à toute comptabilité, ils furent aisés à débiter à Paris pour des particuliers fortunés.

Les errements de la compagnie Chantemerle eurent ainsi de multiples et inattendues conséquences, jusqu'au plus haut niveau. Il est possible que de tels agissements aient été compris par le directeur des Bâtiments du roi comme une rémunération justifiée des services de Tarlé, son contrôleur général, qu'il n'appointa que tardivement. Mais de tels arrangements voire détournements étaient monnaie courante et de bien peu d'importance pour un personnage comme le duc d'Antin, à la tête d'une fortune considérable. En revanche, ils lui permirent d'octroyer aux membres de la cour des marbres fort recherchés, ce qui ne manquait pas d'intérêt. Sa mort, en 1739, fut loin de mettre fin à ces pratiques, mais elles se concentrèrent plus encore autour du monde des marbriers.

Il reste beaucoup à écrire sur un tel sujet, notamment à partir de 1754, lorsque le marquis de Marigny réactiva l'arrêt de 1701 . Il instaura alors une véritable prohibition des marbres qui suscita de multiples fraudes dans les carrières, dans les ports et dans les ateliers et il passa des années à chasser " les abus à détruire ", de Marseille à Bordeaux en passant par les Pyrénées. Mais à ce jeu-là, il semble bien que ce soit à Paris qu'on ait le plus gagné, car on y multipliait les trafics et on y volait des marbres jusque dans les magasins du roi, où pour les évacuer discrètement on cachait des plaques déjà polies - les plus chères - entre des tranches de pierres communes, en les scellant au plâtre et en les maquillant ${ }^{28}$.

De tels procédés prouvent qu'en la matière, l'imagination n'eut pas de limites. Mais au niveau des chantiers, les conséquences en furent-elles toujours négatives? Rien n'est moins certain car si ces détournements se perdirent, financièrement, dans le gouffre des 
dépenses des Bâtiments du roi, matériellement ils ne purent être dissimulés qu'en assurant un approvisionnement suffisant pour ne pas alarmer. Le sérieux de Formont permit de réaliser une grande partie des précieux lambris et pavements de l'Appartement des bains et des Grands appartements, mais la productivité de la compagnie Chantemerle assura quant à elle la fourniture de suffisamment de marbres pour parer à de longues périodes de conflit et subvenir, pour une bonne part, aux escaliers d'honneur, à la Grande galerie et aux salons de la paix et de la Guerre. Et s'il y eut surabondance d'incarnat de Caunes, cela fut loin de nuire à l'harmonie du Grand Trianon ou à la prestance de la Colonnade (ill. 3).

\section{3 : Détail de la colonnade (1686), parc du château de Versailles}

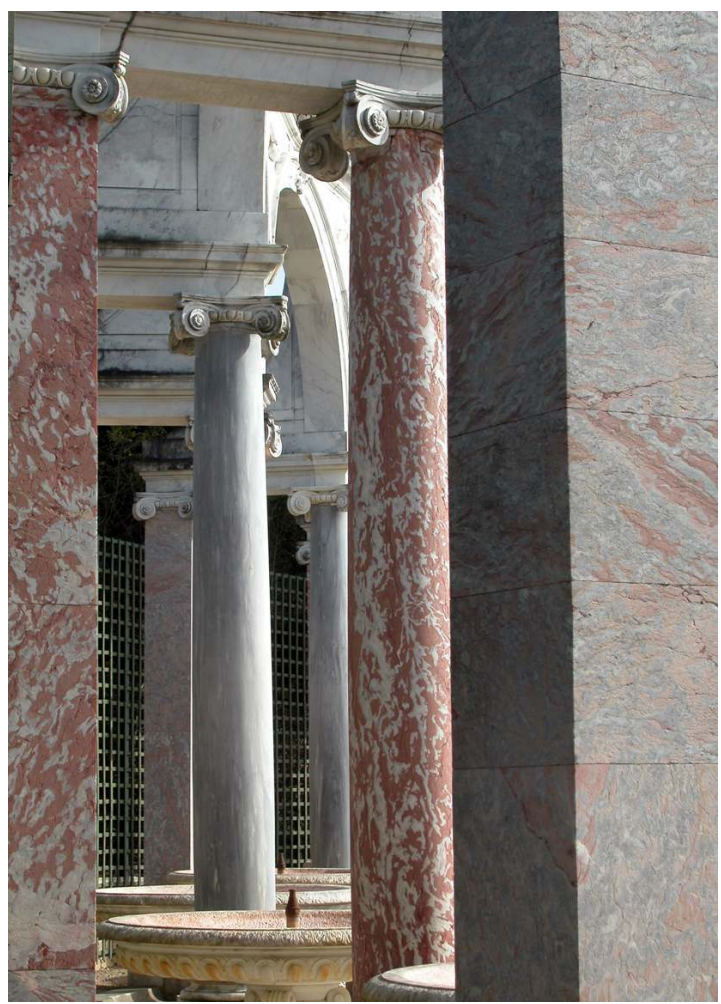

Alternance de piliers en turquin puis en incarnat de Caunes avec des colonnes aussi en incarnat et d'autres en bardiglio de Carrare

Cl. P. Julien

Quant au duc d'Antin, son action fut telle «pour l'honneur de nos Pyrénées » qu'il est certain qu'il eut avant tout le désir de bien faire, tant pour lui que pour le roi. « Nous n'avons besoin que des beaux et non de la quantité » déclarait-il à son intendant, en le poussant à ne rien épargner pour y parvenir. En cela il tranchait singulièrement avec ses prédécesseurs, le Salon d'Hercule en témoigne : Claude-Félix Tarlé y mit en œuvre les plus beaux campan et les plus beaux sarrancolin jamais extraits (ill. 4).

Or, pourtant, ce furent ces marbres-là qui générèrent le plus de profits détournés, pour le bien d'Antin, mais pour la gloire du roi... 


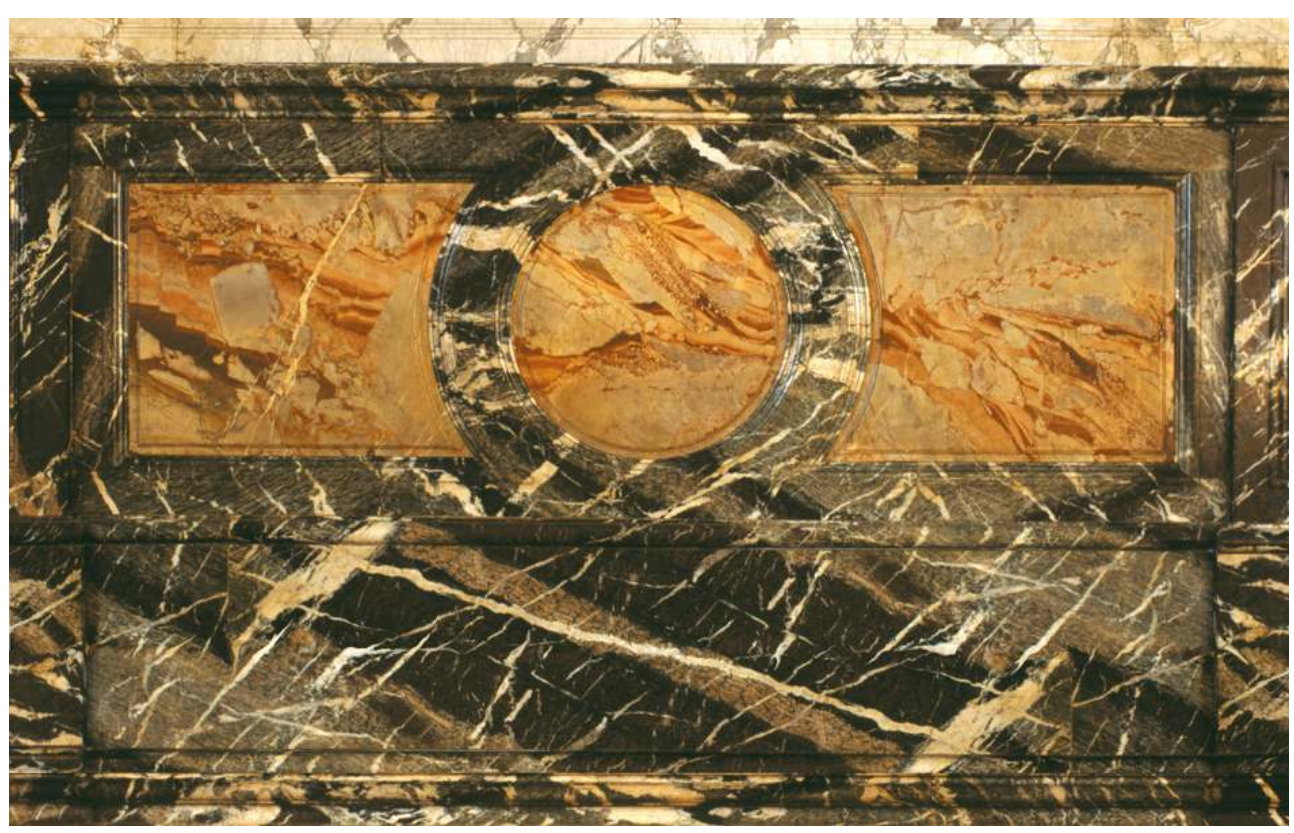

Les panneaux en sarrancolin sont sertis dans de fortes moulures en campan grand mélange

Cl. J.-C. Lepert

\section{NOTES}

1. 1. Geneviève Bresc-Bautier et Hélène du Mesnil, «La politique royale du marbre français (1700-1789) ", Les Ressources minérales et l'histoire de leur exploitation, CTHS, 1986, p. 425-442 ; « Le marbre du roi : l'approvisionnement en marbre de bâtiments du roi, 1660-1715 ", Eighteenth Century Life, "The Art and Architecture of Versailles", 17, 1993, p. 35-54. Geneviève BrescBautier, «Les marbres des Pyrénées sous Louis XIV ", Les marbres blancs des Pyrénées : approches scientifiques et historiques, Saint-Bertrand-de-Comminges, 1995, p. 261-273 ; "L'importation du marbre de Carrare à la cour de Louis XIV : rivalités des marchands et échecs des compagnies », actes du colloque Marbres de Rois, Versailles, 2003, Presses universitaires de Provence, à paraître. Pascal Julien, Marbres : De carrières en palais, du sang des dieux à la gloire des rois, XVI-XVIII ${ }^{e}$ siècles, Manosque, Le Bec-en-l'air, 2006, 270 p.

2. 2. Arch. nat., $\mathrm{O}^{1} 14$, fol. $238 \mathrm{v}^{\mathrm{o}}, 14$ juin 1670 .

3. 3. Arch. nat., $\mathrm{O}^{1}$ 2065. Estat des sommes payées aux cy après nommez pour achapt de marbres pour le roi. Quatre-vingt-un paiements datés et identifiés.

4. 4. Arch. dép. Aude, 3E 14224, fol. 77. Jean-Louis Bonnet, «Des carrières aux marbriers de Caunes-Minervois (XVII ${ }^{\mathrm{e}}$ siècle) », Bulletin de la Société d'Études Scientifiques de l'Aude, XCVIII, 1998, p. 96.

5. 5. Arch. nat., $\mathrm{O}^{1} 2065$. L'association avait été enregistrée le 28 avril 1684 . Il est important d'identifier Jean-Claude Boillot

Boillot, Jean-Claude 
Boileau

avec le « sieur Boileau », car celui-ci, crédité de plusieurs paiements dans les comptes des Bâtiments du roi, a été considéré comme un concurrent de la compagnie Chantemerle Chantemerle, Claude Leseure, sieur de, , alors qu'il en était l'un des associés, ce qui fausse tous les calculs lorsque l'on fait les comptes de cette société. Il est clairement identifié dans l'inventaire après décès de JeanBaptiste Maire, beau-frère de Petit

Petit, Marc-François,

chargé des transports de marbre depuis Toulouse. Arch. dép. Haute-Garonne, 3E 2897, reg. 2, fol. 160-161.

6. 6. Ces quantités, soit 51086 pieds cubes (plus de 5000 tonnes), correspondent sensiblement à celles indiquées par un autre Mémoire des marbres, qui donne la composition des cargaisons embarquées à Bordeaux de juillet 1685 à novembre 1688, toujours sous la surveillance de Martin La différence constatée d'environ 3700 pieds cubes avec un autre compte correspond aux ultimes livraisons faites avant septembre 1689. Arch. nat. $\mathrm{O}^{1} 2075$, Mémoire des marbres tant du Languedoc que des Pirénées partis de Bourdeau pour Rouen suyvant les lettres de voiture enegistrées audit bourdeau. En octobre 1689, 3856 pieds cubes restaient bloqués sur trois navires hollandais immobilisés à Bordeaux.

7. 7. Arch. nat., $\mathrm{O}^{1} 2065,31$ oct. 1689. Toisé et reconnaissance générale des marbres de Languedoc et de Pyrénées qui sont sur les ports du Canal et de Garonne; dont 26946 pieds cubes de caunes, 424 de Saint-Baudery, 1649 de campan et 107 de sarrancolin; soit environ $1034 \mathrm{~m}^{3}$ pour un poids aux alentours de 1000 tonnes.

8. 8. Arch. nat., $\mathrm{O}^{1} 2065$. Les prix étaient progressifs, selon la taille des blocs, 18 livres pour de petits blocs de 3 à 20 pieds cubes jusqu'à 30 livres pour ceux de 40 à 50 p. c. Au renouvellement de ce marché, en 1670, ces prix furent revus à la baisse, hormis pour les marbres des Pyrénées.

9. 9. Arch. nat., $O^{1} 2066$, janvier-mars 1692. Placards et pièces du procès, extrait des registres des juges et consuls des marchands de Paris.

10. 10. Arch. nat., $O^{1} 2066$, lettres de février à septembre 1693.

11. 11. Arch. nat., $\mathrm{O}^{1} 2066,19$ juillet 1693 , lettre de Camuzet à Louvois.

12. 12. La fin de cette société, de plaintes en procès, est des plus complexes. Arch. nat., $\mathrm{O}^{1} 2066,22$ juillet 1693, lettre de Martin

Martin; Michel, ingénieur du roi

à Colbert

Colbert, Jean-Baptiste de, surintendant des bâtiments du roi

de Villacerf.

13. 13. Ange Jacques Gabriel

Gabriel, Jacques-Ange, architecte

, qui le fit recalculer, trouva 549240 livres et plus tard, « il en a esté treuvé par M.

Marigny

Marigny, Abel François, marquis de

$565240 »$.

14. 14. Arch. nat., $\mathrm{O}^{1} 2067,1703$, Récapitulation du toisé de m. de Cotte

Cotte, Robert de, architecte

par luy certifiéle 15 nov. 1703. 
15.

Ce n'est qu'en 1695, après de multiples interventions et remontrances, que l'ordre fut rétabli dans la marbrière de Caunes et les carriers locaux furent contraints de la dégager de tous décombres issus d'exploitations sauvages.

Arch. nat., $\mathrm{O}^{1} 2066$, lettre du 25 décembre 1695.

16. 16. Frédéric Tiberghien a soupçonné du coulage (ce mot d'usage sur les chantiers actuels est ici particulièrement adapté) pour les marbres de Versailles, au sujet de soldats qui dévasèrent des blocs en 1712 à Bordeaux. Mais les faits sont parfois plus complexes qu'ils n'en ont l'air, car cette opération visait à permettre à Tarlé d'inventorier des blocs qui, par la suite seulement, furent source de discrets profits. Frédéric Tiberghien, Versailles, le chantier de Louis XIV, 1662-1715, Perrin, 2002, p. 216.

17. 17. Arch. du château de Valmirande, Marbres, 1712, 17 nov. Lettre du duc d'Antin à Lassus. Les archives des Lassus, Pierre et Marc-Francois, contrôleurs des marbres de 1712 à 1775, contiennent une précieuse correspondance avec les Bâtiments du roi.

18. 18. Pascal Julien, «Le flottage des marbres royaux des Pyrénées à l'Océan », Actes du colloque Forêts et transport. Les modes traditionnels, Paris, ENS, Cahiers d'études 14, 2004, p. 25-29.

19. 19. Arch. du château de Valmirande, Marbres, 17 nov. 1714, lettre du duc d'Antin à Lassus.

20. 20. Arch. du château de Valmirande, Marbres, 23 juin 1715 , lettre du duc d'Antin à Lassus.

21. 21. Pascal Julien, «Pour mieux faire flotter les marbres : Les cartes du "Cours de Garonne" dressées en 1716-1720 par Hippolyte Matis, géographe du roi », Mémoires de la Société archéologique du Midi de la France, 2005, LXV, p. 231-242.

22. 22. Arch. du château de Valmirande, Marbres, 5 avril 1715, lettre du duc d'Antin

Antin, duc d', surintendant des Bâtiments du roi

à Lassus

Pierre de Lassus, contrôleur des marbres du roi

23. 23. Le fait est plaisant car, au moment de l'érection de son marquisat en duché pairie, en mai 1711, il avait été obligé d'échanger certaines terres dont celle de Veyrède, qui devint propriété de Monsieur de Verdelin, qui la revendiqua en 1736 seulement. Il réclama les droits de fortage mais ne récupéra pas ses bois...

24. 24. Jules Guiffrey, Le Duc d'Antin et Louis XIV. Rapports sur l'administration des bâtiments annotés par le Roi, Paris, 1869 , p. 29-30.

25. 25. Arch. nat., $\mathrm{O}^{1} 2068,1737$.

26. 26. Arch. dép. Gironde, C 1749, 3 août 1749, lettre de Tourny à Lenormant.

27. 27. Arch. nat., 012223 (316), 12 août 1723.

28. 28. Arch. nat., $\mathrm{O}^{1} 2085$, Manœuvres qui se pratiquent aux magasins des marbres du roi.

\section{RÉSUMÉS}

La fraude, que l'on a pu parfois considérer comme un profit bien compris, est un phénomène rarement, voire jamais pris en compte dans le cadre des chantiers, alors qu'il s'agit d'une pratique courante et constante dans le monde des métiers, notamment sous l'Ancien Régime. Les marbres, matériaux coûteux à extraire, à transporter et à mettre en œuvre, furent 
particulièrement exposés à ces pratiques, notamment lors de leur emploi à Versailles, où il en fut acheté pour plusieurs millions de livres. De quoi susciter bien des convoitises. La politique marbrière mise en place par les surintendant et directeurs des Bâtiments du roi pour approvisionner ce chantier, de Colbert au duc d'Antin, a laissé la trace de différents abus et de manœuvres menées jusqu'au plus haut niveau, peu apparentes habituellement dans les documents. Leur analyse permet de constater que ce qui apparait parfois comme un phénomène mineur, sinon négligeable, peut avoir d'importantes conséquences sur le long terme, en favorisant parfois des développements inattendus.

Fraud, a phenomenon sometimes regarded simply as a well-understood profit, is seldom taken into account in architectural research, even when it was a common and constant practice among the building trade, especially during the Ancien Régime. It particularly concerned marbles, a costly material to mine, to transport and to employ, chiefly when the authorities bought marble blocks worth millions of livres to be used for Versailles. The order gave ideas to more than one. The policy set out by surintendants and directeurs des Bâtiments du roi to supply the building site in marble, from Colbert to the duc d'Antin, left many marks, usually missing in records, of various violations and intrigues carried even by people in the highest places. Analysing these manoeuvres gives us the opportunity to establish that, what sometimes appears as a minor, if not insignificant, phenomenon can result in long term and occasionally unexpected consequences.

Schmuggelei, öfters als selbstverständlicher Profit geachtet, wurde selten oder fast nie in die Kosten der Baustellen eingerechnet, obwohl es eine übliche und ständige Gewohnheit in der Welt der Handwerker geworden war, besonders unter dem Ancien Régime.Der Gebrauch von wertvollen Materialien wie Marmorsteinen, deren Abbau, Belieferung und Bearbeitung unheimlich teuer waren, konnte bei manchen eine besondere Giersucht erwecken, besonders in Versailles, wo für mehrere Milionen Pfund davon gekauft wurde. Die Beschaffungspolitik des Marmors, so wie sie von den Intendanten und Leitern der Bâtiments du roi (Königsbauten), von Colbert bis zum Herzog von Antin, geführt wurde, hinterließ in den Archiven verschiedene Spuren von Missbrauch und Täuschungsversuchen bis auf höchster Ebene, die gewöhnlich in Archivdokumenten nicht zum Vorschein kommen. Es ergibt sich aus deren Studie, dass ein offensichtlich belangloser Zwischenfall auf lange Zeit beträchtliche Auswirkungen haben konnte und manchmal zu unerwarteter Entwicklung führte.

\section{AUTEUR}

\section{PASCAL JULIEN}

Pascal Julien, professeur d'histoire de l'art moderne à l'université Toulouse-Le-Mirail, et membre de l'UMR 5136-FRAMESPA (France méridionale et Espagne : histoire des sociétés du Moyen Âge à l'époque contemporaine), travaille depuis de nombreuses années sur la sculpture religieuse ainsi que sur les marbres à l'époque moderne. Parmi ses nombreuses contributions dans ces domaines, on trouvera D'ors et de prières. Art et dévotions à Saint-Sernin de Toulouse, XVI ${ }^{e}$-XVIII ${ }^{e}$ siècles aux Presses Universitaires de Provence en 2004 ainsi que Marbres : De carrières en palais, du sang des dieux à la gloire des rois, XVI-XVIII ${ }^{e}$ siècles, aux éditions Le Bec-en-l'air en 2006 pour lequel il a reçu l'année suivante le prix du Cercle Montherlant - Académie des Beaux-Arts 2007 qui récompense un ouvrage de langue française illustré et consacré à l'art. Il a également été récompensé par le prix spécial du jury de l'Académie d'architecture. 